\title{
Nonperturbative renormalisation for low moments of light-meson distribution amplitudes
}

\author{
Jonathan Flynn ${ }^{* a}$, Rudy Arthur ${ }^{b}$, Peter Boyle $^{c}$, Dirk Brömmel ${ }^{d}$, Andreas Jüttner ${ }^{a}$, Chris \\ Sachrajda ${ }^{a}$ and Thomas Rae ${ }^{e}$ \\ ${ }^{a}$ School of Physics and Astronomy, University of Southampton, UK \\ ${ }^{b}$ University of Southern Denmark \\ ${ }^{c}$ School of Physics and Astronomy, University of Edinburgh, UK \\ ${ }^{d}$ Forschungszentrum Jülich, Germany \\ ${ }^{e}$ Institut für Kernphsyik, Johannes-Gutenberg Universität Mainz, Germany \\ E-mail: j.m.flynn@soton.ac.uk, paboyle@ph.ed.ac.uk, juettner@soton.ac.uk, \\ cts@soton.ac.uk, thrae@uni-wuppertal.de
}

\begin{abstract}
We discuss nonperturbative renormalisation of the leading-twist flavour non-singlet operators needed for the calculation of the first and second moments of light-meson distribution amplitudes. On the lattice we use a regularisation-independent symmetric (or non-exceptional) momentum scheme, RI/SMOM, which, for the second moment, allows us to include mixing with a total-derivative operator. We calculate the conversion functions needed to connect the RI/SMOM results to MSbar.
\end{abstract}

The 32nd International Symposium on Lattice Field Theory,

23-28 June, 2014

Columbia University New York, $N Y$

\footnotetext{
*Speaker.
} 


\section{Introduction}

Parton distribution amplitudes (PDAs) are relevant for exclusive QCD processes at large momentum transfers, near the light cone. They provide process-independent nonperturbative information on the bound-state structure of hadrons, in particular the momentum-fraction distribution of partons in a particular Fock state of a hadron. They have been calculated in three main approaches: extraction from experimental form factor data; QCD sum rules; lattice QCD. We are here concerned with the last of these.

Low moments of PDAs can be computed from non-forward local matrix elements with momentum transferred at the operator insertion. For example, for a pseudoscalar meson $P$ the first and second moments $\left\langle\xi^{1}\right\rangle_{P}$ and $\left\langle\xi^{2}\right\rangle_{P}$ are determined by

$$
\begin{gathered}
\left\langle 0\left|S \bar{q}_{a} \gamma_{\mu} \gamma_{5} \stackrel{\leftrightarrow}{D}_{\nu} q_{b}\right| P(p)\right\rangle=\left\langle\xi^{1}\right\rangle_{P} f_{P} S p_{\mu} p_{\nu} \\
\left\langle 0\left|S \bar{q}_{a} \gamma_{\mu} \gamma_{5} \stackrel{\leftrightarrow}{D}_{\nu} \stackrel{\leftrightarrow}{D}_{\rho} q_{b}\right| P(p)\right\rangle=\left\langle\xi^{2}\right\rangle_{P} f_{P} S p_{\mu} p_{\nu} p_{\rho}
\end{gathered}
$$

where $S$ means symmetrised and traceless in Lorentz indices Bare lattice operators need renormalisation and matching to a continuum scheme like $\overline{\mathrm{MS}}$. For the second moment, because there is non-zero momentum transfer, there will be mixing of the double-covariant-derivative operator with a double-total-derivative operator,

$$
S \bar{q}_{a} \gamma_{\mu} \gamma_{5} \stackrel{\leftrightarrow}{D_{\nu}} \stackrel{\leftrightarrow}{D} q_{\sigma} \quad \text { and } \quad S \partial_{\mu} \partial_{\nu}\left(\bar{q}_{a} \gamma_{\sigma} \gamma_{5} q_{b}\right)
$$

Hence, on the lattice we will need to compute

$$
\left\langle\xi^{1}\right\rangle^{\overline{\mathrm{MS}}}=\frac{Z_{D, D}}{Z_{A}}\left\langle\xi^{1}\right\rangle^{\text {bare }} \quad \text { and } \quad\left\langle\xi^{2}\right\rangle^{\overline{\mathrm{MS}}}=\frac{Z_{D D, D D}}{Z_{A}}\left\langle\xi^{2}\right\rangle^{\text {bare }}+\frac{Z_{D D, \partial \partial}}{Z_{A}}
$$

where $Z_{D, D}, Z_{D D, D D}$ and $Z_{D D, \partial \partial}$ are renormalisation constants to be determined, ideally nonperturbatively $\left(Z_{A}\right.$ is the renormalisation constant for the light-quark axial vector current, which we determine elsewhere [1]).

\section{Previous RBC/UKQCD calculation}

In our previous work ([2,3] and in preparation), we used two lattice spacings, $a^{-1}=1.73 \mathrm{GeV}$ and $2.28 \mathrm{GeV}$. Figure 1 shows results for the kaon 1 st moment and pion 2 nd moment. The kaon 1 st moment has little lattice spacing dependence, while there is more visible $a$-dependence for the pion second moment. However, in these calculations the mixing with the double total-derivative operator is perturbative [3], since our previous nonperturbative renormalisation was performed in the $\mathrm{RI}^{\prime} / \mathrm{MOM}$ scheme (see below) with zero momentum transfer at the operator. Where we had both, the perturbative and nonperturbative renormalisation constants differed, as shown in table 1.

\section{Nonperturbative renormalisation}

We use a Rome-Southampton regularisation independent (RI) momentum subtraction (MOM) scheme. For operator $O$, the renormalisation constant $Z_{O}$ is determined by

$$
\Lambda_{R}^{O}=\frac{1}{Z_{q}} Z_{O} \Lambda_{B}^{O}
$$



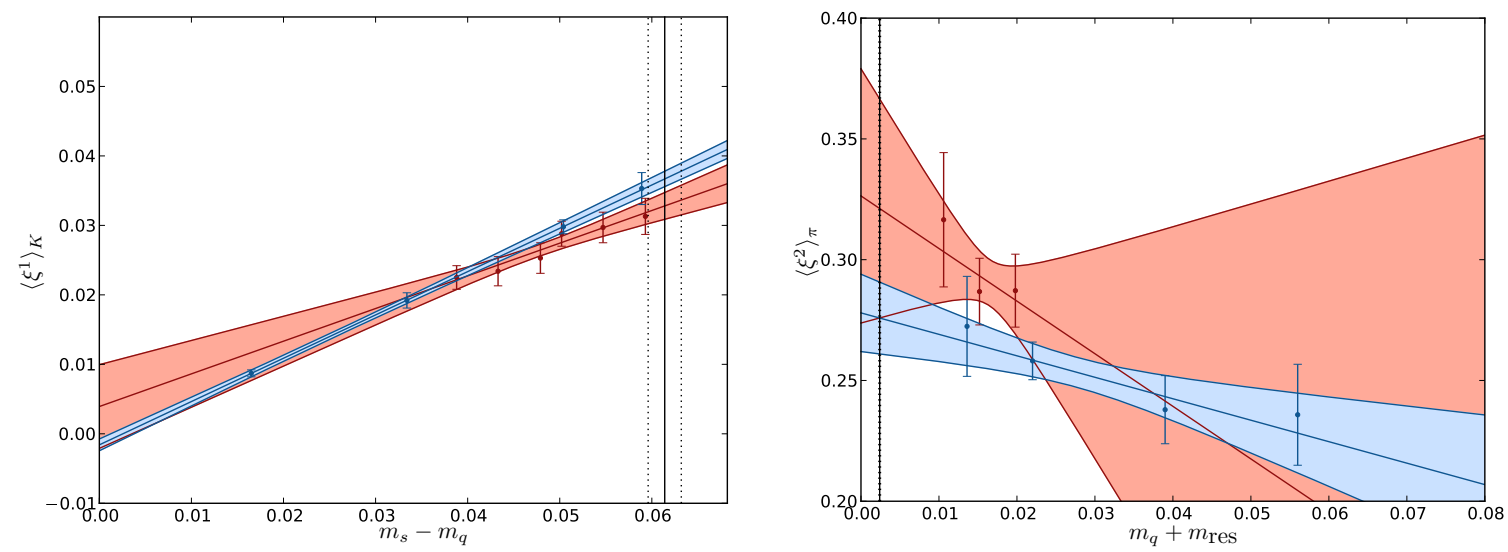

Figure 1: Chiral extrapolations of results for the kaon 1st moment, left, and pion 2nd moment, right, at two different lattice spacings: $a^{-1}=1.73 \mathrm{GeV}$ blue, $2.28 \mathrm{GeV}$ red. The 1 st moment result, with nonperturbative renormalisation, shows little lattice-spacing dependence. For the 2nd moment, where the double covariant derivative operator is nonperturbatively renormalised but the total-derivative operator is perturbatively renormalised, there is more $a$-dependence.

\begin{tabular}{llll} 
& $Z_{D, D} / Z_{A} Z_{D D, D D} / Z_{A} Z_{D D, \partial \partial} / Z_{A}$ \\
\hline nonperturbative & $1.50(2)$ & $1.97(5)$ & - \\
mean-field imp PT & $1.28(4)$ & $1.51(6)$ & $0.015(4)$
\end{tabular}

Table 1: Perturbative and nonperturbative renormalisation constants on the $a^{-1}=2.28 \mathrm{GeV}$ lattice with $\overline{\mathrm{MS}}$ scale $\mu=2 \mathrm{GeV}$.

where $\Lambda^{O}$ is an amputated quark two-point function with an insertion of $O$ (Lorentz indices implicit). The subscripts $R$ and $B$ denote renormalised and bare respectively. For operators which mix, as is the case for the 2 nd moment PDA calculation, $Z_{O}$ will be a matrix. $Z_{q}$ is the quark wavefunction renormalisation constant.

We impose the renormalisation condition (or conditions for operators which mix) at a particular momentum configuration with associated scale $\mu$, satisfying $\Lambda_{\mathrm{QCD}} \ll \mu \ll 1 / a$. In the SMOM or symmetric momentum scheme, in Euclidean space, the incoming quark momentum $q$, incoming antiquark momentum $p$ and momentum transfer $q+p$ satisfy

$$
q^{2}=p^{2}=(q+p)^{2}=\mu^{2}, \quad q \cdot p=-\mu^{2} / 2
$$

as indicated in figure 2.

Previously we used the RI'/MOM scheme with an exceptional momentum choice $q^{2}=p^{2}=\mu^{2}$ and no momentum transfer, $q+p=0$. The SMOM scheme allows mixing with total derivative operators (needed in our case), suppresses contamination from IR effects and is expected to be better-behaved (or at least not worse behaved) in the perturbative series needed for conversion to the $\overline{\mathrm{MS}}$ scheme. Here we are concerned with the conversion functions from SMOM to $\overline{\mathrm{MS}}$ for the PDA 1st and 2nd moments. Evaluating those conversions is done in the continuum and the relevant 


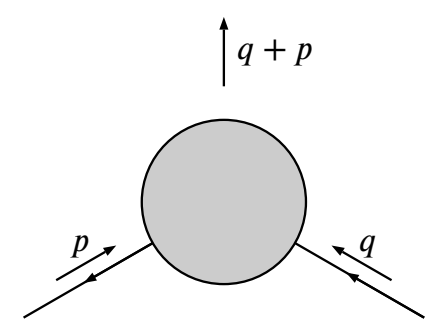

Figure 2: Momentum configuration for the SMOM scheme applied to a quark two-point function with operator insertion.

calculations have been performed by J Gracey to 3 loops in $\overline{\mathrm{MS}}$ and to 2 loops in SMOM $[4,5,6,7]$. The amputated two-point quark Green functions are matrices in colour and spin indices and the choice of how those are traced into the scalars $\Lambda_{R, B}^{O}$ will be reflected in the precise values of the conversion functions.

\section{Operator basis and scalar coefficients}

We use the following basis of $C$-eigenstate operators ${ }^{1}$ :

$$
\begin{array}{rlrl}
X_{2} & =S \bar{\psi} \gamma_{\mu} \stackrel{\leftrightarrow}{D}_{\nu} \psi & X_{3} & =S \bar{\psi} \gamma_{\mu} \stackrel{\leftrightarrow}{D}_{\nu} \stackrel{\leftrightarrow}{D}_{\sigma} \psi \\
\partial X_{2} & =S \partial_{\mu}\left(\bar{\psi} \gamma_{\nu} \psi\right) & \partial \partial X_{3} & =S \partial_{\mu} \partial_{\nu}\left(\bar{\psi} \gamma_{\sigma} \psi\right) \\
\partial X_{3} & =S \partial_{\mu}\left(\bar{\psi} \gamma_{\nu} \stackrel{\leftrightarrow}{D}_{\sigma} \psi\right)
\end{array}
$$

$X_{2}, \partial X_{2}, \partial X_{3}$ are all multiplicatively renormalised while $X_{3}$ and $\partial \partial X_{3}$ mix. For the PDA 1 st and 2nd moments we need to renormalise $X_{2}, X_{3}$ and $\partial \partial X_{3}$, but we include the single total-derivative operators $\left(\partial X_{2,3}\right)$ in order to make some checks: $\partial X_{2}$ and $\partial \partial X_{3}$ are total derivatives of vector current and should have the same anomalous dimension; similarly, $\partial X_{3}$ is a total derivative of $X_{2}$.

Following Gracey [4, 5, 6, 7], we expand amputated quark two-point Green functions of these operators in bases of Lorentz tensor structures with scalar coefficients. For example, for the first moment operators (suppressing spin and colour indices):

$$
\begin{aligned}
\Lambda^{\mu \nu}(p, q)_{\mathrm{sym}} & =\sum_{i=1}^{10} P_{(i)}^{\mu \nu}(p, q) \Sigma_{i}\left(\mu^{2}\right) \\
\Sigma_{i}\left(\mu^{2}\right) & =\frac{1}{\mu^{2}} \operatorname{Tr}\left[M_{i j} P_{(j)}^{\mu \nu}(p, q) \Lambda_{\mu \nu}(p, q)_{\mathrm{sym}}\right]
\end{aligned}
$$

where 'sym' means evaluated at an SMOM symmetric momentum configuration and $P_{(i)}^{\mu v}(p, q)$ are 10 Lorentz tensors with

$$
N_{i j}=\frac{1}{\mu^{2}} \operatorname{Tr}\left[P_{(i)}^{\mu \nu} P_{(j) \mu \nu}\right]_{\mathrm{sym}} \quad M=N^{-1}
$$

${ }^{1}$ Operators with and without $\gamma_{5}$ renormalise in the same way if chiral symmetry is respected. Our lattice simulations use a domain wall fermion action with good chiral symmetry properties. 
There are similar decompositions for bilinear and second moment operators with 6 and 14 Lorentz structures respectively.

Gracey used a different basis of operators. Changing to the $C$-conserving basis above leads to relations between Gracey's $\overline{\mathrm{MS}}$ anomalous dimensions which are all satisfied ${ }^{2}$. For the amputated Green functions, charge-conjugation implies a set of relations between the scalar coefficients in our basis. These are satisfied by the Gracey continuum calculations (after the change of basis) and by lattice data for a unit gauge field. They are also well-satisfied by our lattice data at the two lattice spacings.

\section{SMOM renormalisation conditions}

A specific SMOM renormalisation scheme is fixed by demanding that after tracing with some 'projector' $P$, the renormalised amputated Green function should give the tree-level result

$$
\frac{1}{Z_{q}} \operatorname{Tr}\left(Z_{O} \Lambda_{B, \text { sym }}^{O} P\right)=\operatorname{Tr}\left(\Lambda_{\text {tree,sym }}^{O} P\right)
$$

We aim to choose $P$ 's to respect the charge-conjugation properties of the operators and, for operators which are total derivatives of vector current, to maintain the Ward identity.

For example, the SMOM renormalisation condition for the vector current [8]

$$
\frac{1}{12 \mu^{2}} \frac{Z_{V}}{Z_{q}} \operatorname{Tr}\left(k_{\mu} \Lambda_{V, B}^{\mu} \not k\right)=1 \quad \text { where } \quad k=q+p
$$

maintains the Ward identity $k_{\mu} \Lambda_{V, R}^{\mu}=S_{R}^{-1}(-p)-S_{R}^{-1}(q)$, where $S_{R}$ is the renormalised quark propagator. We choose renormalisation conditions for total derivatives of the vector current

$$
\begin{aligned}
\frac{Z_{\partial X_{2}}}{Z_{q}} \operatorname{Tr}\left[\left(S k_{\mu} k_{\nu}\right) \not k \Lambda_{\partial X_{2}, B}^{\mu \nu}\right] & =9 i\left(\mu^{2}\right)^{2} \\
\frac{Z_{\partial \partial X_{3}}}{Z_{q}} \operatorname{Tr}\left[\left(S k_{\mu} k_{\nu} k_{\rho}\right) \not k \Lambda_{\partial \partial X_{3}, B}^{\mu \nu \rho}\right] & =-6\left(\mu^{2}\right)^{3}
\end{aligned}
$$

and confirm that the conversion functions from SMOM to $\overline{\mathrm{MS}}$ for all three operators are then 1.

As another example, for the second moment the operator $X_{3}=S \bar{\psi} \gamma_{\mu} \stackrel{\leftrightarrow}{D} \stackrel{\leftrightarrow}{D_{\sigma}} \psi$ mixes with $\partial \partial X_{3}=S \partial_{\mu} \partial_{\nu}\left(\bar{\psi} \gamma_{\sigma} \psi\right)$. The tree-level matrix elements are

$$
\begin{aligned}
& \Lambda_{\mu \nu \sigma}^{\stackrel{\leftrightarrow}{D D}}(p, q)_{\text {tree }}=-S\left(q_{\mu}-p_{\mu}\right)\left(q_{\nu}-p_{\nu}\right) \gamma_{\sigma}=\frac{\mu^{2}}{3}\left(P_{3, \mu \nu \sigma}+P_{1, \mu \nu \sigma}-P_{2, \mu \nu \sigma}\right) \\
& \Lambda_{\mu \nu \sigma}^{\partial \partial}(p, q)_{\text {tree }}=-S\left(q_{\mu}+p_{\mu}\right)\left(q_{\nu}+p_{\nu}\right) \gamma_{\sigma}=\frac{\mu^{2}}{3}\left(P_{3, \mu \nu \sigma}+P_{1, \mu \nu \sigma}+P_{2, \mu \nu \sigma}\right)
\end{aligned}
$$

where $P_{1,2,3}$ are three of 14 possible Lorentz structures. To fix the renormalisation constants $Z_{D D, D D}$ and $Z_{D D, \partial \partial}$ to get from the bare lattice results to SMOM, we impose renormalisation conditions

$$
\begin{aligned}
\frac{1}{Z_{q}} \operatorname{Tr}\left[\left((M P)_{3}+(M P)_{1}-(M P)_{2}\right)\left(Z_{D D, D D} \Lambda_{B}^{\stackrel{\leftrightarrow}{D D}}+Z_{D D, \partial \partial} \Lambda_{B}^{\partial \partial}\right)\right] \\
=\operatorname{Tr}\left[\left((M P)_{3}+(M P)_{1}-(M P)_{2}\right) \Lambda_{\text {tree }}^{\stackrel{\leftrightarrow \leftrightarrow \leftrightarrow}{D D}}\right]=\mu^{2}
\end{aligned}
$$

\footnotetext{
${ }^{2}$ In fact they determine one of the second moment anomalous dimensions to one higher power in $g^{2}$. In the notation of [7], the relation $\gamma_{11}^{W_{3}}+\gamma_{12}^{W_{3}}-\gamma_{22}^{W_{3}}=0$ fixes the $\left(g^{2}\right)^{3}$ term in $\gamma_{12}^{W_{3}}$.
} 


$$
\begin{array}{r}
\frac{1}{Z_{q}} \operatorname{Tr}\left[\left((M P)_{3}+(M P)_{1}+(M P)_{2}\right)\left(Z_{D D, D D} \Lambda_{B}^{\stackrel{\leftrightarrow \leftrightarrow}{D D}}+Z_{D D, \partial d} \Lambda_{B}^{\partial \partial}\right)\right] \\
=\operatorname{Tr}\left[\left((M P)_{3}+(M P)_{1}+(M P)_{2}\right) \Lambda_{\text {tree }}^{\stackrel{\leftrightarrow \leftrightarrow \leftrightarrow}{D D}}\right]=\frac{\mu^{2}}{3}
\end{array}
$$

In these expressions the trace is on spin and colour indices. There is also a summation on the (suppressed) Lorentz indices. The notation $(M P)_{i}$ denotes the summation $M_{i j} P_{(j)}$ where $M$ is the matrix defined in equation 4.1.

\section{Conversion functions}

Having specified SMOM renormalisation conditions, we need to evaluate the conversion functions to give final results in $\overline{\mathrm{MS}}$. Suppose operators renormalised in SMOM and $\overline{\mathrm{MS}}$ are related by a (matrix) $C$,

$$
O_{\overline{\mathrm{MS}}}=C O_{R}
$$

The corresponding relation for amputated two-point quark Green functions with an insertion of $O$ is

$$
\Lambda_{\overline{\mathrm{MS}}}=\frac{1}{C_{q}} C \Lambda_{R} \quad \text { where } \quad C_{q} \equiv \frac{Z_{q, \overline{\mathrm{MS}}}}{Z_{q}}
$$

Expand the Green function $\Lambda_{a}$ for operator $O_{a}$ in terms of tensors $P_{i}$ with scalar coefficients $\Sigma_{a i}$

$$
\Lambda_{a}=\sum_{i} \Sigma_{a i} P_{i} \quad \Sigma_{a i}=\operatorname{Tr}\left[(M P)_{i} \Lambda_{a}\right]
$$

Our renormalisation prescription is that tracing $\Lambda_{R a}$ with some projector $P_{A}$ gives the tree-level (or other chosen) result, $T_{a A}$

$$
\operatorname{Tr}\left(\Lambda_{R a} P_{A}\right)=T_{a A}
$$

We may need to choose several $P_{A}$ 's if the operators mix. Let $N_{i A}^{P} \equiv \operatorname{Tr}\left(P_{i} P_{A}\right)$ and use $\Lambda_{R}=$ $C_{q} C^{-1} \Lambda_{\overline{\mathrm{MS}}}$ to write

$$
C_{q} C_{a b}^{-1} \Sigma_{b i}^{\overline{\mathrm{MS}}} N_{i A}^{P}=T_{a A}
$$

Gracey's $\overline{\mathrm{MS}}$ results give the scalar coefficients $\Sigma^{\overline{\mathrm{MS}}}$ while $N^{P}$ and $T$ (and $C_{q}$ ) are also known. We can then impose enough conditions to solve for the elements of $C$. Subsequently, combining $C$ with the $Z$ 's determined by our SMOM renormalisation conditions allows us to convert from lattice to $\overline{\mathrm{MS}}$ at scale $\mu$. We can then use $\overline{\mathrm{MS}}$ anomalous dimensions to scale to a common value, say $2 \mathrm{GeV}$.

Once an SMOM renormalisation prescription has been fixed, the SMOM anomalous dimensions can be found from

$$
\gamma_{\mathrm{SMOM}}=C^{-1} \gamma_{\overline{\mathrm{MS}}} C-\mu \frac{d C^{-1}}{d \mu} C
$$

We close by presenting the conversion functions calculated for our choice of SMOM renormalisation prescription. Here $a=g^{2} / 16 \pi^{2}, \alpha$ is the gauge parameter (which will be set to 0 since our lattice Green functions are evaluated in Landau gauge) and $N_{f}$ is the number of flavours. For the first moment the non-zero elements of $C$ are

$$
\begin{aligned}
& C_{11}=1-(1.63903 \alpha+5.12484) a-\left(3.8244 \alpha^{2}+6.37866 \alpha-12.1458 N_{f}+106.359\right) a^{2} \\
& C_{22}=1
\end{aligned}
$$


The subscript indices 1 and 2 refer to $X_{2}$ and $\partial X_{2}$ respectively.

For the second moment the non-zero elements are

$$
\begin{aligned}
& C_{11}=1-(2.18537 \alpha+8.24516) a-\left(5.18357 \alpha^{2}+2.38666 \alpha-19.8008 N_{f}+156.444\right) a^{2} \\
& C_{12}=(0.138749 \alpha+1.15755) a+\left(0.419338 \alpha^{2}+1.95065 \alpha-2.31945 N_{f}+20.0837\right) a^{2} \\
& C_{22}=1 \\
& C_{33}=1-(1.63903 \alpha+5.12484) a-\left(3.8244 \alpha^{2}+6.37866 \alpha-12.1458 N_{f}+106.359\right) a^{2}
\end{aligned}
$$

where now the indices 1,2 and 3 refer to $X_{3}, \partial \partial X_{3}$ and $\partial X_{3}$ respectively. The $C_{i j}$ are 1 for operators which are total derivatives of the vector current. We also observe that $\partial X_{3}$ is a total derivative of $X_{2}$ and has the same conversion coefficient (compare $C_{11}$ for the first moment with $C_{33}$ for the second moment).

\section{Summary}

We are interested in calculating 1st and 2nd moments of PDAs with fully nonperturbative renormalisation. This involves non-forward matrix elements, allowing mixing with total derivative operators and demanding the use of an SMOM renormalisation scheme. Continuum calculations exist $[4,5,6,7]$ to allow the needed conversion functions from SMOM to $\overline{\mathrm{MS}}$ to be computed once renormalisation conditions have been imposed. This enables a fully nonperturbative lattice computation with continuum perturbation theory needed only for the final conversion to $\overline{\mathrm{MS}}$.

\section{References}

[1] RBC/UKQCD Collaboration, Y. Aoki et. al., Continuum Limit Physics from $2+1$ Flavor Domain Wall QCD, Phys.Rev. D83 (2011) 074508 [1011. 0892].

[2] R. Arthur, P. Boyle, D. Brommel, M. Donnellan, J. Flynn et. al., Light Meson Distribution Amplitudes, PoS LATTICE2010 (2010) 154 [1011. 2883].

[3] R. Arthur, P. Boyle, D. Brömmel, M. Donnellan, J. Flynn et. al., Lattice Results for Low Moments of Light Meson Distribution Amplitudes, Phys.Rev. D83 (2011) 074505 [1011. 5906].

[4] J. Gracey, RI'/SMOM scheme amplitudes for deep inelastic scattering operators at one loop in QCD, Phys.Rev. D83 (2011) 054024 [1009.3895].

[5] J. Gracey, RI'/SMOM scheme amplitudes for quark currents at two loops, Eur.Phys.J. C71 (2011) 1567 [1101.5266].

[6] J. Gracey, Two loop renormalization of the $n=2$ Wilson operator in the RI'/SMOM scheme, JHEP 1103 (2011) 109 [1103. 2055].

[7] J. Gracey, Amplitudes for the $n=3$ moment of the Wilson operator at two loops in the RI'/SMOM scheme, Phys.Rev. D84 (2011) 016002 [1105.2138].

[8] C. Sturm, Y. Aoki, N. Christ, T. Izubuchi, C. Sachrajda et. al., Renormalization of quark bilinear operators in a momentum-subtraction scheme with a nonexceptional subtraction point, Phys.Rev. D80 (2009) 014501 [0901.2599]. 